\title{
1 Quantifying cumulative phenotypic and genomic evidence for procedural generation of
} 2 metabolic network reconstructions

\section{Thomas J. Moutinho Jr. ${ }^{1}$, Benjamin C. Neubert ${ }^{1}$, Matthew L. Jenior ${ }^{1}$, Jason A. Papin ${ }^{1}$}

$4 \quad{ }^{1}$ University of Virginia, Department of Biomedical Engineering

\section{Abstract}

Genome-scale metabolic network reconstructions (GENREs) are valuable tools for understanding microbial community metabolism. The process of automatically generating GENREs includes identifying metabolic reactions supported by sufficient genomic evidence to generate a draft metabolic network. The draft GENRE is then gapfilled with additional reactions in order to recapitulate specific growth phenotypes as indicated with associated experimental data. Previous methods have implemented absolute mapping thresholds for the reactions automatically included in draft GENREs; however, there is growing evidence that integrating annotation evidence in a continuous form can improve model accuracy. There is a need for flexibility in the structure of GENREs to better account for uncertainty in biological data, unknown regulatory mechanisms, and context specificity associated with data inputs. To address this issue, we present a novel method that provides a framework for quantifying combined genomic, biochemical, and phenotypic evidence for each biochemical reaction during automated GENRE construction. Our method, Constraint-based Analysis Yielding reaction Usage across metabolic Networks

19 (CANYUNs), generates accurate GENREs with a quantitative metric for the cumulative evidence for each reaction included in the network. The structure of a CANYUN GENRE allows for the simultaneous

21 integration of three data inputs while maintaining all supporting evidence for biochemical reactions that

22 may be active in an organism. CANYUNs is designed to maximize the utility of experimental and

23 annotation datasets and to ultimately assist in the curation of the reference datasets used for the

24 automatic reconstruction of metabolic networks. We validated CANYUNs by generating an E. coli K-12

25 model and compared it to the manually curated reconstruction iML1515. Finally, we demonstrated the 
bioRxiv preprint doi: https://doi.org/10.1101/2021.08.13.456222; this version posted August 13, 2021. The copyright holder for this preprint (which was not certified by peer review) is the author/funder, who has granted bioRxiv a license to display the preprint in perpetuity. It is made available under aCC-BY 4.0 International license. use of CANYUNs to build a model by generating an E. coli Nissle CANYUN GENRE using novel phenotypic

27 data that we collected. This method may address key challenges for the procedural construction of metabolic networks by leveraging uncertainty and redundancy in biological data. 


\section{Introduction}

Complex microbial communities play an important role in human physiology (1-5). Genome-

32 scale metabolic network reconstructions (GENREs) have been shown to model the functional capabilities of microbes and their interactions in communities $(6,7)$. A GENRE is a constraint-based model structure that enables the combination of various forms of biological data to gain an improved mechanistic understanding of metabolism (8). This form of modeling explicitly accounts for biochemical thermodynamics and stoichiometry to represent the physical constraints that govern cellular metabolism. Methods used to generate GENREs are progressively being automated to reduce time and resource requirements with the goal of modeling the vast number of unique species and strains that reside in human associated microbiota (9-12). However, there remains a need for advancements in the procedural generation of GENREs to improve the accounting of uncertainty in the source biological data.

The foundational data that procedurally generated GENREs are built upon is a universal biochemical reaction network with associated reference genetic annotation data for sequence-toreaction mapping. When building an organism-specific GENRE, a genome is annotated with precise biochemical reactions. The annotation process typically involves a threshold of sequence alignment that is used to determine if a sequence is similar enough to the reference sequence to justify annotation with the associated biochemical function (13). The data used to build GENREs is incomplete and subject to uncertainty, necessitating gapfilling of the metabolic network generated via genetic data alone. Gapfilling is the process of adding biochemical reactions with low or no evidence to a GENRE based on functional phenotypic growth data and the cellular biomass requirements. The resulting accuracy of the curated GENRE is then calculated by how well it recapitulates the phenotypic growth data utilized for training. There are additional methods for further assessment of model quality involving other data types, such as gene essentiality data, and separate validation data (14). Recent methods have 
demonstrated that utilizing gene annotation alignment scores in a continuous way can help to improve

54 62 justify inclusion. gapfilling results $(10,15)$.

A curated GENRE consists of a set of reactions that have biological evidence suggesting that they are catalyzed by the organism. When a GENRE is procedurally generated the remaining error is commonly dominated by false growth calls; these errors indicate that these models over-predict the metabolic capabilities of an organism. Additionally, annotation alignment scores, universal biochemical network source data, and annotation reference data are all often left out from published GENREs $(16,17)$. Without the source sequence-to-reaction data used to generate a GENRE, the reactions that are included in the curated GENRE lack explicit indication of what type of biological evidence was used to

Phenotypic growth data used for gapfilling is not utilized the same as context specific data is treated when used to contextualize a GENRE. For example, transcriptomics data can be used to gain insight into how metabolic flux might be routed in an unknown growth condition $(18,19)$. Phenotypic growth data provides a similar type of context-specific data compared to transcriptomic data, yet it is utilized to build a metabolic network rather than contextualize the existing universal metabolic network. From a biological point of view, it is an over simplification of an organism-specific metabolic network to ignore the existence of gene regulation during the GENRE building process (20). The practice of including all genetic data in the functional GENRE and then gapfilling remaining essential reactions with phenotypic data results in an over-constrained assessment of the biological system under investigation (10). There is a need for additional flexibility in the structure of GENREs to better account for uncertainty in biological data, unknown regulatory mechanisms, and context specificity associated with data inputs.

In this study, we present a novel method for contextualizing a manually curated universal metabolic network through the simultaneous integration of genetic annotation data and phenotypic 
growth data. Our method, Constraint-based Analysis Yielding reaction Usage across metabolic Networks

77 (CANYUNs), procedurally generates a GENRE by explicitly quantifying the combined biological evidence for the inclusion of reactions in the resulting network. CANYUNs utilizes a continuous weighting for each reaction in a curated universal metabolic network to quantify the evidence provided by the biological data that is used during the reconstruction process. Rather than gapfilling a draft network by leveraging phenotypic data, CANYUNs determines the reactions required for computational growth in each known growth condition separately to quantify the cumulative evidence for each reaction. The cumulative evidence generated for each reaction during the CANYUNs training process is subsequently used to determine the reactions that are included in the final GENRE. The resulting CANYUNs model consists of the universal metabolic network and associated reference annotations, organism-specific genetic alignment scores, phenotypic growth data, and certainty values associated with each reaction included

87 in the curated network.

\section{Results}

\section{Constraint-based Analysis Yielding reaction Usage across metabolic Networks (CANYUNs)}

The model training process in CANYUNs is designed to capture and quantify the cumulative future model curation. 
100

101

102

103

104

105

106

107

108

109

metabolite formulas did not match, we used the iML1515 formulas to maximize the number of massbalanced reactions in the final universal network. For additional curation, we used an optimization method to check the network for generation of free-mass (see Methods). In short, we created intracellular sink reactions for each intracellular metabolite in the network and closed all exchange reactions to ensure the network did not have access to any extracellular metabolites. We then maximized the sum of flux through all sink reactions to identify any metabolites produced due to massimbalanced reactions or mass-generating loops. We curated the universal network by manually removing reactions that were contributing to free-mass generation. Using this optimization-based method, we were able to more rigorously identify free-mass generation in the network compared to simply checking each reaction for mass-balance.

We utilized BLASTp to align the genome of the target organism with reference sequences in the CarveMe sequence-to-reaction dataset. We used the sequence alignment bitscores for $E$. coli K-12 genes and the CarveMe dataset to then generate reaction bitscores using the published method (10). We subsequently used a step-wise linear transformation to convert the reaction bitscores to reaction weights that fall between -1 and 1 to use during linear optimization and flux balance analysis. We developed a novel formulation of flux balance analysis called, Data Guided Flux Balance Analysis (dgFBA) specifically for CANYUNs. This optimization equation minimizes the sum of flux through all reactions with low or no genetic evidence while simultaneously maximizing the sum of flux through all reactions with substantial genetic evidence. The degree to which a reaction is minimized or maximized is linearly determined by the reaction weights. During a dgFBA optimization, flux is required through the biomass reaction to represent growth. Importantly, dgFBA allowed us to determine the flux-carrying reactions (FCRs) in each experimental growth condition by setting the exchange reactions to represent the specific growth media conditions. By tracking the FCRs for each growth condition, we were able to then calculate the ratio of growth conditions in which a reaction carries flux and determine reaction Certainty 
124 Values (CVs) for each FCR indicating confidence in the presence of each biochemical function in the

125 target organism.

In the final stage, all flux-carrying reactions across the experimental growth conditions are used

127 to generate an organism-specific CANYUNs model (Figure 1A). The resulting network is processed

128 further by selectively removing a single reaction, or a small set of reactions, to further improve the

129 overall accuracy of the model and adjust the type of error remaining. For validation of CANYUNs, we

130 generated an automatic GENRE for E. coli K-12 leveraging phenotypic nutrient utilization data obtained

131 from EcoCyc and compared it to iML1515 (21-23). 
A

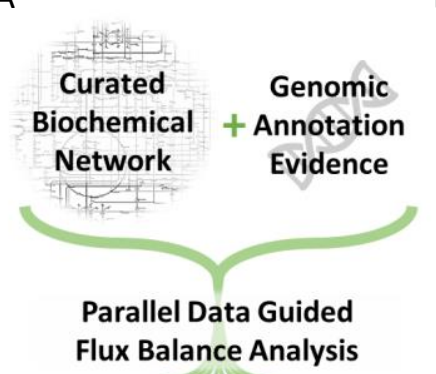

Flux Balance Analysis

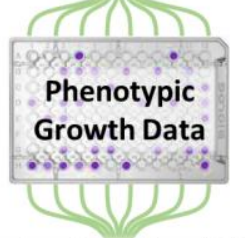

Reaction Certainty Values and conditionally essential reactions for model curation

CANYUNs Model
B $\frac{\text { Universal Biochemical }}{\text { Network }}$

Reactions:

Exchange: 529

Intracellular: 4140

Metabolites:

Exchange: 533

Intracellular: 1501

Periplasmic: 573

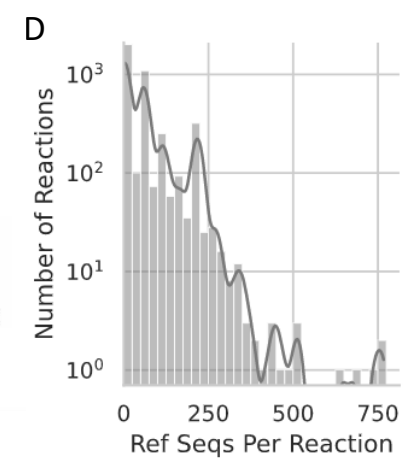

C

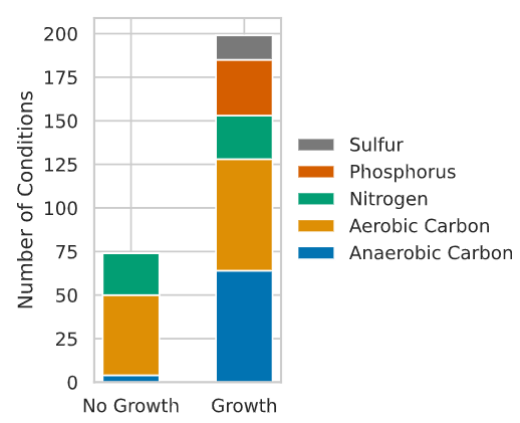

E

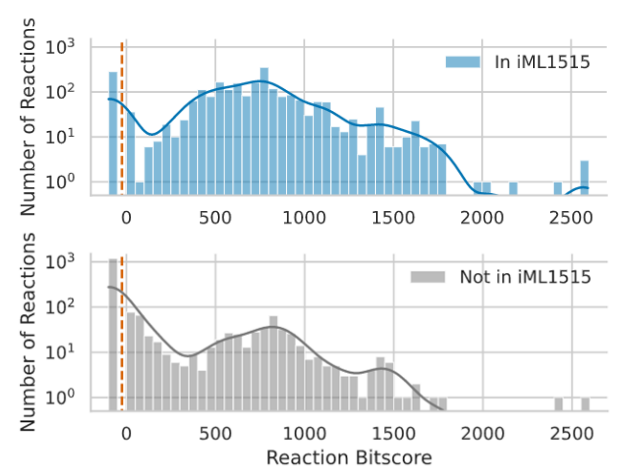

Figure 1: The CANYUNs pipeline integrates genomic, phenotypic, and biochemical data to quantitatively identify reactions that are likely catalyzed by an organism. A) Genomic annotation data and phenotypic growth data for a specific organism are used to influence the flux distribution through a curated universal biochemical network to build an organism-specific metabolic network model. Parallel growth simulations using Data Guided Flux Balance Analysis for each known experimental growth condition allows for a model building process that is not influenced by the order in which growth conditions are integrated. This process allows for the explicit quantification of reaction Certainty Values, determined by the ratio of times a reaction carries flux across all of the condition-specific solutions to the total number of conditions. B) The universal biochemical network used in this study consists of reactions from the CarveMe dataset as well as novel reactions added from the manually curated E. coli metabolic network, iML1515. C) The phenotypic data used in this study includes Biolog minimal media growth data from $\sim 275$ different conditions. $D$ ) The sequence to reaction dataset used to calculate reaction annotation evidence consists of over 4,000 reactions with 1 to 800 sequences associated with each reaction. E) The distribution of reaction bitscores for $E$. coli $\mathrm{K}-12$ shows that there are reactions in the universal network with high evidence that are not included in iML1515. There are also many reactions with low evidence that are not included in iML1515, as expected. The annotation evidence generated for $E$. coli K-12 shows that there are 1,460 reactions in the universal biochemical network that have no genetic evidence associated with them, 260 of these reactions are in iML1515 and 1,200 of them are not.

Data Guided Flux Balance Analysis

The reaction bitscores for $E$. coli $\mathrm{K}-12$ were calculated directly from BLASTp sequence alignment 
a bitscore of 0 and the rest range from 1 to 2,500 (Figure 2A). A typical bitscore threshold for assigning a

137 reference enzymatic metabolic function to the query sequence(s) is between 200 and 500 (24-26). The

138 level of confidence in a functional call increases with the value of the bitscore, yet small changes in a

139 sequence can result in large functional changes. Bitscores below the threshold also contribute

140 information about the protein in question, values that are just shy of the threshold may still have the

141 same function as the reference protein; however, scores that fall far short of the threshold suggest that

142 the protein in question does not have the function of the reference.

We designed dgFBA to account for some of the uncertainty inherent in setting a threshold for

144 assigning function to a given protein by utilizing reaction weights that are a function of the reaction

145 bitscores. The reaction weights influence the reactions that carry flux in the optimization solution.

146 Genomic annotation data must be transformed to a range of values that are compatible with dgFBA. The

147 transformation function used in this study is graphically displayed in Figure 2B. This function can be

148 adjusted based on the user's preferences. In this study we selected a bitscore threshold of 500 based on

149 a sensitivity analysis that demonstrated that model accuracy was insensitive to values between 200 and

150500 (Figure 2B).

FCRs in a dgFBA solution are a result of complex interactions among the reaction weight values,

152 media condition, and flux demands (i.e. biomass). Flux is maximized through reactions with a bitscore

153 above 500 and minimized through reactions below 500 . However, the degree of maximization and

154 minimization depend upon the value of the bitscore. The low evidence reactions that are included in the

155 final flux solution are likely essential for flux through biomass and can be thought of as gapfilled

156 reactions that maintain their genomic annotation evidence (Figure 2E and 2F). Utilizing the reaction

157 bitscores in this way allows for additional flexibility with reactions near to the bitscore threshold of 500

158 where the reaction weight is equal to zero. Reactions with weights near to zero are much less impacted 

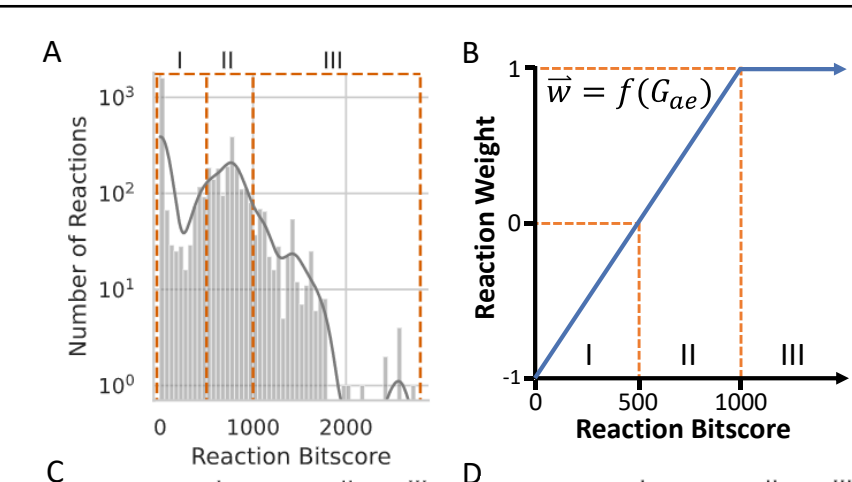

$\operatorname{dgF} \boldsymbol{B A}$
Maximize: $\sum \vec{w} * \vec{v}$

D

$\mathrm{F}$

subject to:
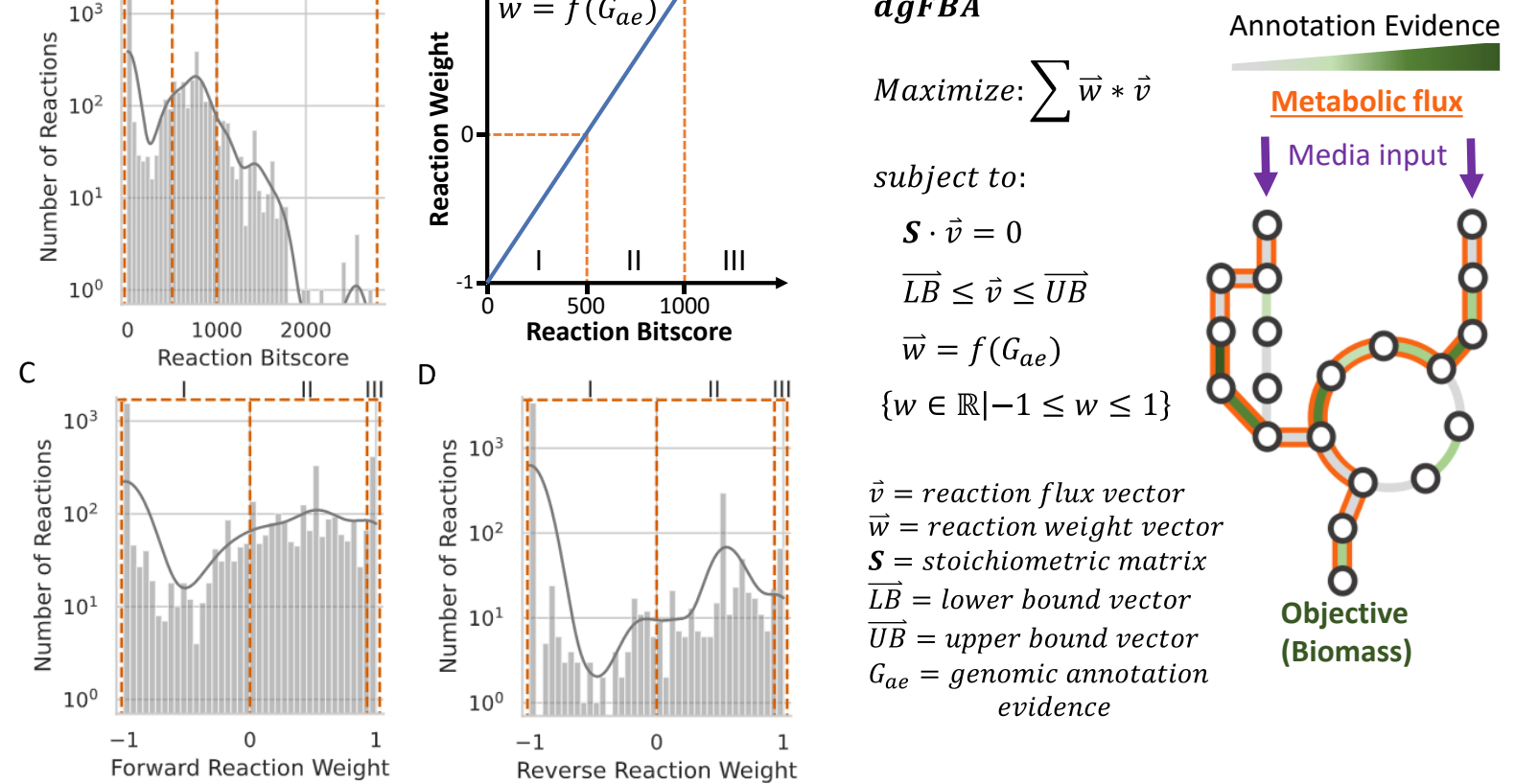

$$
\begin{aligned}
& \boldsymbol{S} \cdot \vec{v}=0 \\
& \overrightarrow{L B} \leq \vec{v} \leq \overrightarrow{U B} \\
& \vec{w}=f\left(G_{a e}\right)
\end{aligned}
$$

$\{w \in \mathbb{R} \mid-1 \leq w \leq 1\}$

$\vec{v}=$ reaction flux vector

$\vec{w}=$ reaction weight vector

$\boldsymbol{S}=$ stoichiometric matrix

$\overrightarrow{L B}=$ lower bound vector

$\overrightarrow{U B}=$ upper bound vector

$G_{a e}=$ genomic annotation

$\downarrow$ Media input $\downarrow$ evidence

Figure 2: Data Guided Flux Balance Analysis. A) Distribution of reaction bitscores for E. coli K-12. B) This is a visual representation of the transformation function for calculating the reaction weights based on reaction bitscores. The reaction bitscore of 500 corresponds with zero in the weight space. C) Distribution of the calculated weights for forward reactions. D) The distribution of weights for reverse reactions shows that there are far fewer reactions that allow flux in both directions or only in the reverse direction. E) Data Guided Flux Balance Analysis optimization problem. Reactions with a positive weight are maximized and reactions with a negative weight are minimized proportional to the value of the weight. F) Toy network example demonstrating the flux-carrying reactions that would result from the pictured annotation evidence distribution and media inputs.

Data Guided Flux Balance Analysis can be compared to parsimonious enzyme usage flux balance analysis (pFBA) to demonstrate how flux through the network changes with additional layers of information. The objective of pFBA is to uniformly minimize the sum of flux across all reactions, while maintaining flux through the biomass reaction (27). Since dgFBA maximizes the weighted flux through reactions with genetic evidence the flux distribution is consistently different from the pFBA flux distribution. However, the two optimization problems remain similar because the majority of reactions 


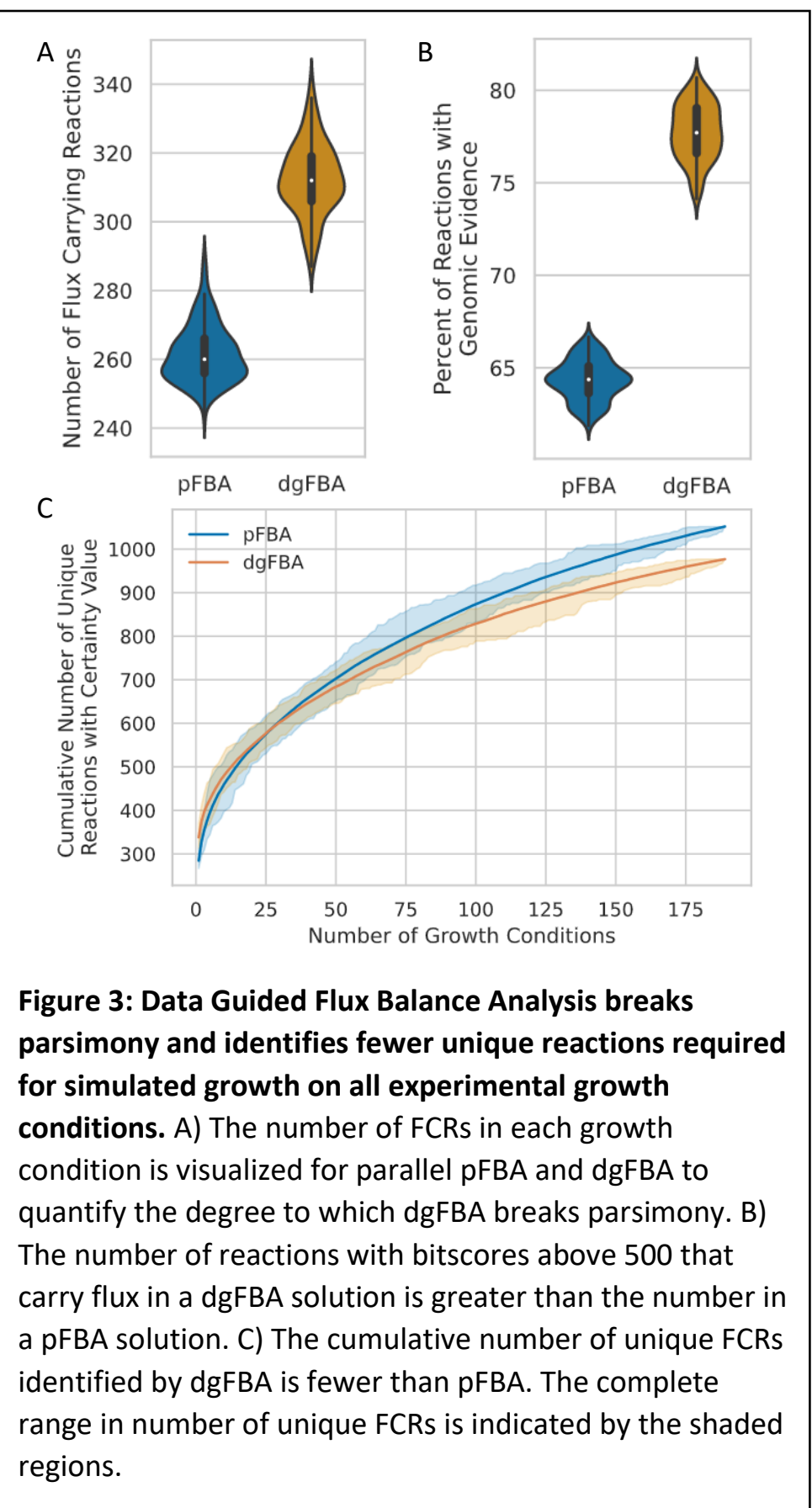

187 in the universal network do not have

genetic evidence and are thus

minimized in a dgFBA problem, just as

they are in a pFBA problem. We

compared dgFBA to pFBA to quantify

how much impact the genetic data has

on the flux distribution for each

solution. We generated a separate

pFBA and dgFBA flux solution for each

known E. coli K-12 growth condition.

We used two metrics to verify that

dgFBA results in more FCRs, while also

increasing the number of reactions that

have associated annotation evidence

(Figure $3 \mathrm{~A}$ and $3 \mathrm{~B}$ ). We found that

across all 199 growth conditions, the

average number of FCRs for dgFBA was

305 , which was 45 reactions greater

than the average for pFBA solutions.

We expected dgFBA to identify less

parsimonious solutions than pFBA due to the influence imparted on the solution from the annotation evidence. It is important to note that the number of FCRs for the dgFBA solution in a single condition was always greater than the number of FCRs in the corresponding pFBA solution by at least $10 \mathrm{FCRs}$. A 
192

proportion of FCRs that have associated annotation evidence. We found that the average number of FCRs with annotation evidence greater than or equal to our reaction bitscore threshold of 500 in dgFBA solutions was nearly $20 \%$ greater than the pFBA solutions.

To assess FCRs across all of the known growth conditions, we generated rarefaction curves each consisting of 10,000 samples to measure the full distribution of unique permutations of growth conditions that could be used to generate the GENRE. The x-axis displays the number of growth conditions used to calculate the total number of unique FCRs found (Figure $3 \mathrm{C}$ ). The shaded regions show the minimum and maximum values sampled for each number of conditions included. The small range between the minimum and maximum indicates that there is minimal advantage to optimizing for the minimum number of growth conditions that provide the maximum training value. Each individual growth condition adds unique reactions to the cumulative set of unique FCRs. However, the asymptotic shape of the average curves indicates that the total number of valuable unique minimal growth conditions may not be far beyond 200 conditions. The number of unique FCRs identified by dgFBA across all growth conditions is fewer than pFBA, indicating that there is a core set of FCRs with genetic evidence that dgFBA preferentially identifies over pFBA (Figure 3C). These data indicate that dgFBA performs as intended, reactions with genetic evidence preferentially carry flux even when there is a more parsimonious path which results in a diversion of flux away from extraneous reactions that are more parsimonious but lack sufficient genetic evidence.

\section{Certainty Values Determine the Reactions that are Included in the CANYUN GENRE for E. coli K-12}

The CANYUNs pipeline involves generating a dgFBA solution for each of the known growth conditions using the curated universal metabolic network. During the process of recording the FCRs for each condition, the directionality of each flux value is used to specifically determine the cumulative evidence for each reaction specific to direction. We calculated a reaction certainty value for each reaction in the universal metabolic network based on the set of FCRs from each growth condition. The 
Certainty Value (CV) for a reaction is the ratio of the number of times the reaction carries flux in the

217 known growth conditions over the total number of known growth conditions. A CV indicates the

218 cumulative experimental evidence for the presence of the biochemical function in an organism-specific

219 metabolic network. Using the E. coli K-12 genome and phenome, we calculated 690 reactions with CVs

220 greater than zero in the forward direction (Figure 4A), and 127 reactions with CVs greater than zero in

221 the reverse direction (Figure 4B). There are 45 FCRs that were found to have both forward and reverse

222 reaction CVs (Figure 4C).

We built an E. coli K-12 specific GENRE that consists of the reactions identified to have CVs

224 greater than zero, including only the reaction directionalities specifically with CVs. Reversible reactions

225 that receive a CV above zero in only one direction were set to only allow flux in that direction. We

226 simulated growth in each of the known growth conditions using the resulting cumulative model to

227 determine the baseline performance. The draft metabolic network reconstruction, at this point, had an

228 overall accuracy of $80 \%$ with a strong bias toward false positive growth calls (Figure 4D). To improve the

229 model accuracy, we calculated the conditionally essential reactions for each of the conditions predicted

230 to allow for growth, including false growth predictions. A comparison across the sets of conditionally

231 essential reactions revealed reactions that, when removed, would provide a net benefit for improving

232 the overall accuracy of the adjusted model. We identified that with the removal of a single reaction,

233 RuBisCO, the number of false positives was reduced by 38 conditions and the number of true positives

234 was only decreased by 7 conditions. RuBisCO was manually selected for removal because it had the

235 maximum net benefit of 31 conditions and the least annotation evidence. All of the other candidate

236 reactions for removal are plotted based on their net benefit to accuracy upon removal versus their

237 annotation evidence value (Figure 4E). This process could be repeated for further alteration of the

238 model. Although RuBisCO is an obvious reaction that should not have been included in the universal

239 metabolic network before generating reaction CVs, this result demonstrates that there are reactions 
bioRxiv preprint doi: https://doi.org/10.1101/2021.08.13.456222; this version posted August 13, 2021. The copyright holder for this preprint (which was not certified by peer review) is the author/funder, who has granted bioRxiv a license to display the preprint in perpetuity. It is made available under aCC-BY 4.0 International license.

240 that may require manual removal from the universal metabolic network based on additional biological

241 knowledge aside from a lack of annotation evidence or contribution to mass-generating loops. However,

242 CANYUNs allowed for rapid identification of reactions that may be improperly included during the

243 process of procedural generation of a CANYUN GENRE. 


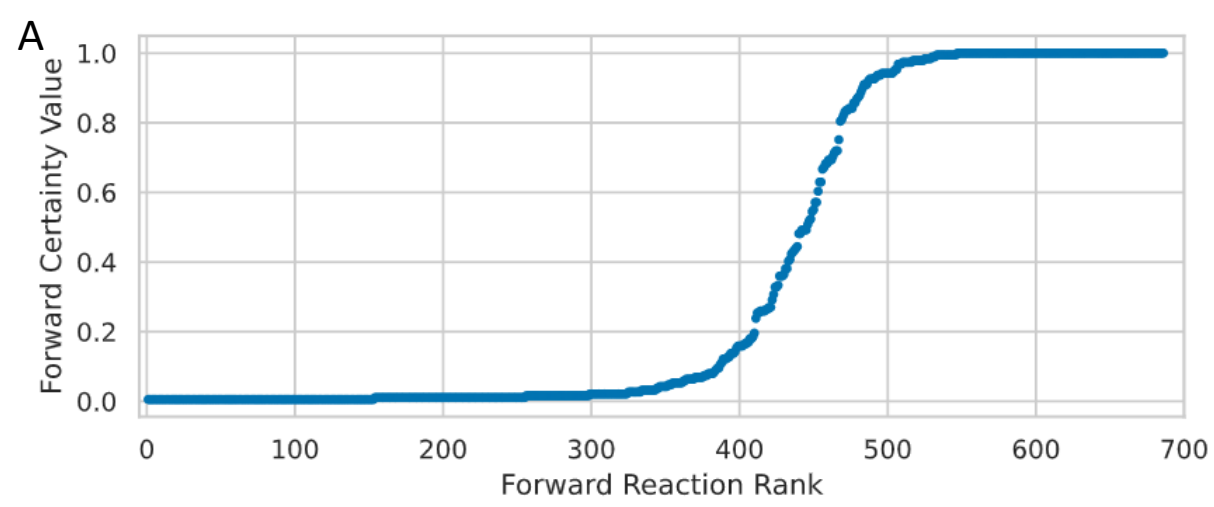

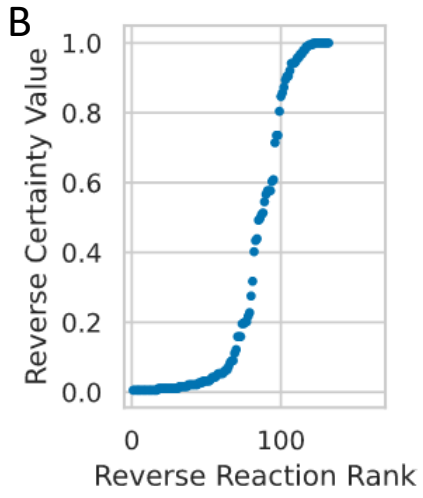

D

\begin{tabular}{lcc}
\multicolumn{3}{c}{ Initial CANYUN Model } \\
\hline & $\begin{array}{l}\text { Predicted } \\
\text { Growth }\end{array}$ & $\begin{array}{l}\text { Predicted No } \\
\text { Growth }\end{array}$ \\
\hline $\begin{array}{l}\text { Exp. } \\
\text { Growth }\end{array}$ & True Positive & False Negative \\
Exp. No & False Positive & True Negative \\
Growth & 51 & 23 \\
\hline
\end{tabular}

Overall accuracy $80 \%$

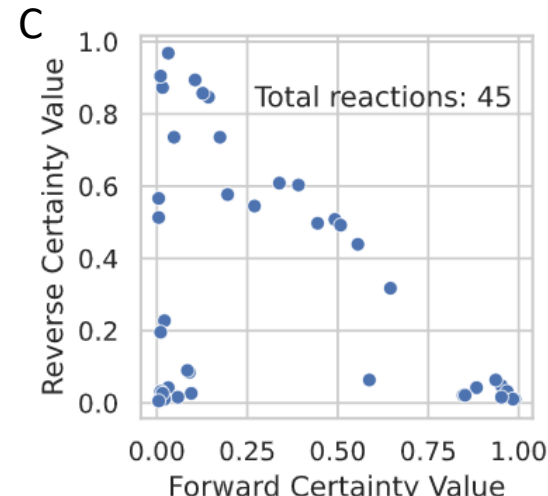

E

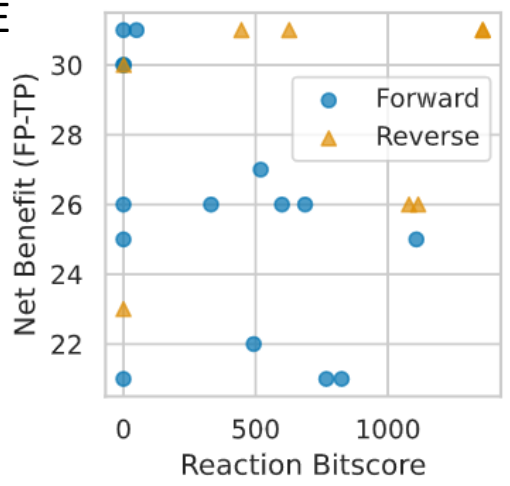

Figure 4: E. coli K-12 CANYUNs model generation and draft processing. A) Ranked scatter plot of forward reaction Certainty Values. B) Reverse reaction Certainty Values. C) Certainty values for reversible reactions that carry flux in both directions. D) Initial accuracy of CANYUNs model before processing. E) Conditionally essential reactions allow for the user to identify reactions that can be selectively removed from the resulting model that improve the overall predictive accuracy. The net benefit refers to the number of false positives that will be correct minus the number of true positives lost due to removing a given reaction. RuBisCO is the forward reaction in the top left corner of the plot with maximum net benefit and minimum genetic evidence. 
The final E. coli K-12 model from the CANYUNs pipeline can be compared with two automatically

247 generated models using CarveMe and the manually curated model iML1515 to benchmark and validate

248 its performance. Using the same input biochemical and genetic data as the CANYUNs model, we

249 generated a CarveMe model without using phenotypic data to establish how subsequent gapfilling

250 impacts the model accuracy (Figure 5A). The gapfilled CarveMe model that we generated had an overall

251 accuracy of $76 \%$, a $24 \%$ improvement over the untrained model (Figure 5B). The training process results

252 in nearly all of the false negative predictions being corrected, as can be expected. The manually curated

253 reconstruction, iML1515, was not specifically curated for all of the known growth conditions used to

254 train the CarveMe model and the CANYUN model, but it remains a valuable point of comparison as the

255 best representation of E. coli K-12 metabolism that is currently available. Our E. coli K-12 CANYUNs

256 model shows the highest overall accuracy, while maintaining a balance in type 1 and type 2 error. The

257 distinction between false positives and false negatives is notable because false negatives represent an

258 opportunity to selectively add organism-specific reactions to the universal model that directly corrects

259 the issue. However, correcting false positive errors involves finding reactions to remove or adjust that

260 result in minimal negative impacts to the rest of the network. CANYUNs provides a method for

261 selectively adjusting the balance of error based on user preferences during the construction of the

262 GENRE. 


\begin{tabular}{|c|c|c|c|c|c|}
\hline \multirow[t]{2}{*}{ Carv } & \multicolumn{2}{|c|}{ CarveMe Model without Gapfilling } & \multirow[t]{2}{*}{ B } & \multicolumn{2}{|c|}{ CarveMe Gapfilled Model } \\
\hline & $\begin{array}{l}\text { Predicted } \\
\text { Growth }\end{array}$ & $\begin{array}{l}\text { Predicted No } \\
\text { Growth }\end{array}$ & & $\begin{array}{l}\text { Predicted } \\
\text { Growth }\end{array}$ & $\begin{array}{l}\text { Predicted No } \\
\text { Growth }\end{array}$ \\
\hline $\begin{array}{l}\text { Exp. } \\
\text { Growth }\end{array}$ & $\begin{array}{c}\text { True Positive } \\
100\end{array}$ & $\begin{array}{l}\text { False Negative } \\
96\end{array}$ & $\begin{array}{l}\text { Exp. } \\
\text { Growth }\end{array}$ & $\begin{array}{c}\text { True Positive } \\
197\end{array}$ & $\begin{array}{c}\text { False Negative } \\
3\end{array}$ \\
\hline $\begin{array}{l}\text { Exp. No } \\
\text { Growth }\end{array}$ & $\begin{array}{c}\text { False Positive } \\
30 \\
\end{array}$ & $\begin{array}{c}\text { True Negative } \\
39 \\
\end{array}$ & $\begin{array}{l}\text { Exp. No } \\
\text { Growth }\end{array}$ & $\begin{array}{c}\text { False Positive } \\
62 \\
\end{array}$ & $\begin{array}{c}\text { True Negative } \\
12 \\
\end{array}$ \\
\hline \multicolumn{3}{|c|}{ Overall accuracy $52 \%$} & \multicolumn{3}{|c|}{ Overall accuracy $76 \%$} \\
\hline \multirow[t]{2}{*}{ C } & \multicolumn{2}{|l|}{ iML1515 } & \multicolumn{3}{|c|}{ CANYUN Model } \\
\hline & $\begin{array}{l}\text { Predicted } \\
\text { Growth }\end{array}$ & $\begin{array}{l}\text { Predicted No } \\
\text { Growth }\end{array}$ & & $\begin{array}{l}\text { Predicted } \\
\text { Growth }\end{array}$ & $\begin{array}{l}\text { Predicted No } \\
\text { Growth }\end{array}$ \\
\hline $\begin{array}{l}\text { Exp. } \\
\text { Growth }\end{array}$ & $\begin{array}{c}\text { True Positive } \\
158\end{array}$ & $\begin{array}{l}\text { False Negative } \\
41\end{array}$ & $\begin{array}{l}\text { Exp. } \\
\text { Growth }\end{array}$ & $\begin{array}{c}\text { True Positive } \\
189\end{array}$ & $\begin{array}{c}\text { False Negative } \\
10\end{array}$ \\
\hline $\begin{array}{l}\text { Exp. No } \\
\text { Growth }\end{array}$ & $\begin{array}{l}\text { False Positive } \\
28\end{array}$ & $\begin{array}{c}\text { True Negative } \\
46\end{array}$ & $\begin{array}{l}\text { Exp. No } \\
\text { Growth }\end{array}$ & $\begin{array}{c}\text { False Positive } \\
13\end{array}$ & $\begin{array}{c}\text { True Negative } \\
61\end{array}$ \\
\hline \multicolumn{3}{|c|}{ Overall accuracy $75 \%$} & \multicolumn{3}{|c|}{ Overall accuracy $92 \%$} \\
\hline \multicolumn{6}{|c|}{$\begin{array}{l}\text { Figure 5: The } \text { E. coli CANYUNs GENRE performs better than iML1515 and CarveMe when simulating } \\
\text { growth on all known phenotypic data. A) The CarveMe model without gapfilling has a base accuracy of } \\
52 \% \text {. B) The CarveMe model we trained using all of the phenotypic data performs with an accuracy of } \\
76 \% \text {. However, there is a strong bias toward false positive predictions. C) The manually curated E. coli } \\
\text { K-12 model, iML1515, was not trained using all of the growth conditions. However, it performs with } \\
75 \% \text { accuracy while maintaining a relatively even split between false positive predictions and false } \\
\text { negative predictions. D) The CANYUNs model we generated performs with } 92 \% \text { accuracy. The increased } \\
\text { accuracy is primarily due to an improvement in true negative prediction rate. }\end{array}$} \\
\hline
\end{tabular}

\section{CANYUNs more accurately identifies the reactions present in iML1515}

It is possible to generate a CANYUNs model using pFBA instead of dgFBA; in this case no genetic data is incorporated to influence the flux distribution of the solution for each growth condition. The

267 most parsimonious solution is determined for each condition when using pFBA. In doing so, we are able

268 to establish a more precise understanding of how the inclusion of genetic annotation evidence impacts

269 the discovery of reactions when compared to the manually curated E. coli K-12 model, iML1515. We did

270 not expect the CANYUNs reactions to align perfectly with iML1515 since the manual curation process did

271 not include all of the growth conditions used to train the CANYUNs model. However, since the 
272 sequence-to-reaction dataset used to generate annotation evidence does not include all of the

273 sequences used to build iML1515, we were able to track the FCRs that CANYUNs identifies without

274 annotation evidence, yet are confirmed to be $E$. coli K-12 reactions by the iML1515 model. The 'Likely

275 additions' category (Figure 6A and 6B) represents a set of reactions from the CANYUNs model with high

276 genetic annotation evidence (bitscore above 500) that are not present in iML1515 and cannot be

277 validated using this comparison, but they may represent reactions that could be added to iML1515 to

278 improve alignment with the phenotypic data. We demonstrate that the dgFBA CANYUN model has $12 \%$

279 greater alignment with iML1515 at the reaction level, compared to the pFBA CANYUN model (Figure 6C).

280 The discovery accuracy is calculated as the number of FCRs that are identified by CANYUNs while lacking

281 sufficient annotation evidence yet that were included in the iML1515 model. The dgFBA CANYUN model

282 has a discovery accuracy of $62 \%, 24 \%$ greater than the pFBA CANYUN model. CANYUNs accurately

283 identifies reactions that should be included in the $E$. coli K-12 metabolic network validated by the most

284 recent manually curated reconstruction, iML1515. 


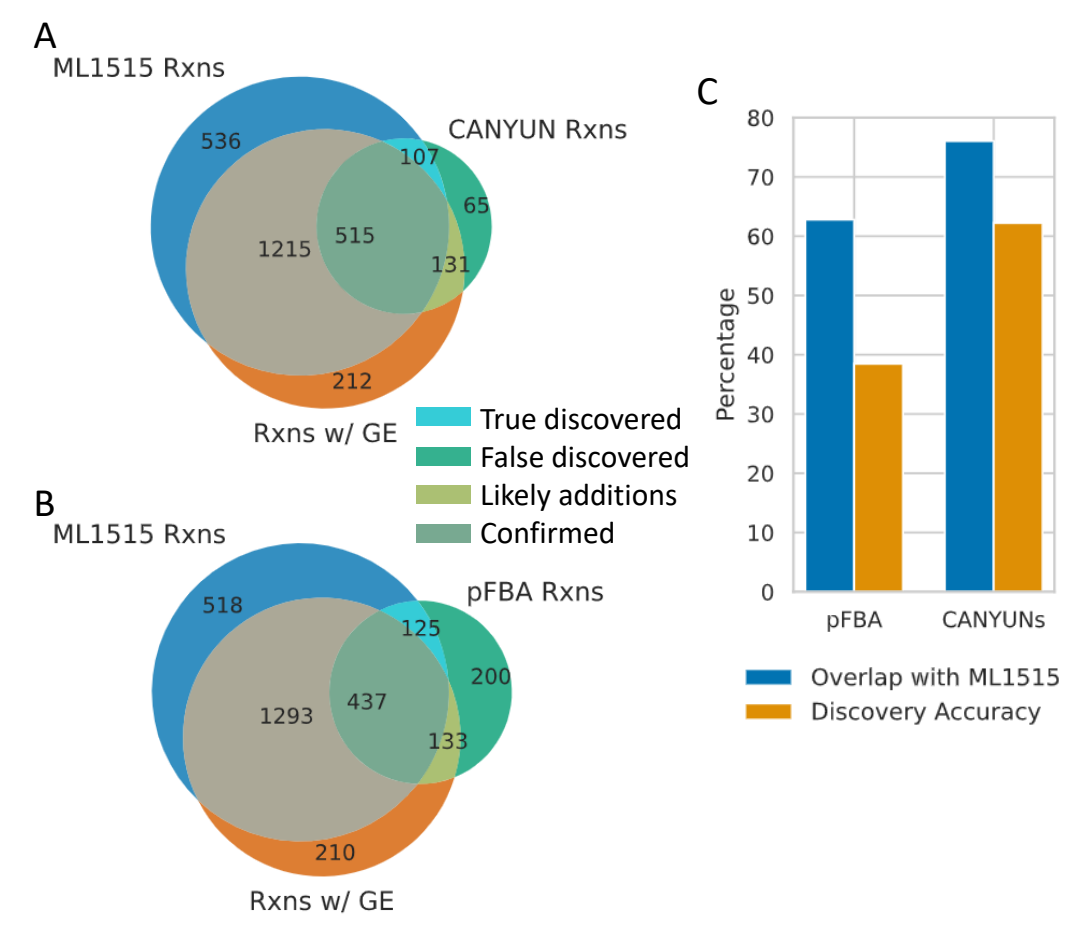

Figure 6: CANYUNs reaction Certainty Values accurately identify reactions found in iML1515.

The manually curated metabolic network, iML1515, provides a point of comparison to determine if CANYUNs accurately identifies reactions for inclusion in the network. A) By comparing the CANYUNs model with iML1515, we were able to place reactions into four categories. FCRs with genetic evidence and in iML1515 (confirmed), FCRs without genetic evidence in iML1515 (true discovered), FCRs with genetic evidence not in iML1515 (likely additions), and FCRs without genetic evidence and not in iML1515 (false discovered). The total amount of genetic evidence that is used to generate a CANYUNs model influences the accuracy of the FCRs. B) When we use pFBA instead of dgFBA in the CANYUNs pipeline, there are far more reactions that lack genetic evidence and are not in iML1515. C) The percent overlap of FCRs with reactions present in iML1515 increases from 62\% when no genetic evidence is used ( $p F B A$ ) to $76 \%$ overlap when all of the available genetic evidence is used.

A further analysis of the reaction CVs demonstrates that the accuracy of reaction inclusion in a

iML1515 improves rapidly when the bottom 30 reactions with the lowest certainty values are ignored.

Overall, dgFBA provides a noticeable benefit over pFBA; however, there is a set of about 50 core 

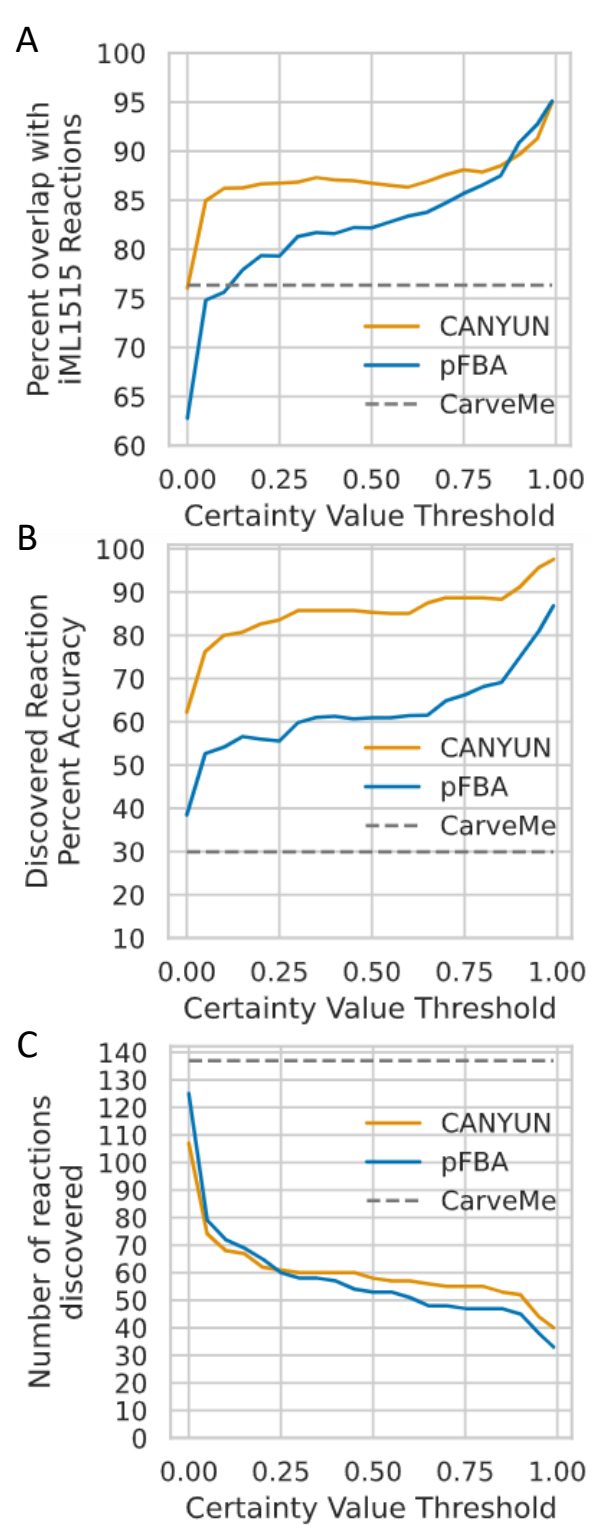

Figure 7: Reaction Certainty Values correlate with accurate reaction inclusion and comparison with CarveMe. A) The percentage of reactions identified by CANYUNs that align with the iML1515 model correlates with the associated certainty value. All reactions with a certainty value greater than or equal to 0.99 have a $94 \%$ chance of being in the iML1515 model. B) The accuracy of discovered reactions, confirmed by iML1515, increases with the certainty values assigned using CANYUNs. C) Although the accuracy of the discovered reactions increases with the certainty value, there is a significant drop in the number of reactions with the increase. outperforms pFBA and CarveMe

when evaluating the discovery

accuracy. The performance of

CANYUNs is in part explained by the

reduced total number of discovered

reactions compared to CarveMe.

That number represents a

significant advancement when

considering the process of manually

validating the reactions with

insufficient annotation evidence by

searching for the appropriate gene-

protein rule to add to the

sequence-to-reaction dataset.

\section{CANYUN GENRE for the probiotic} strain: $E$. coli Nissle

We built a novel model of

the E. coli Nissle metabolic network

to demonstrate the application of

CANYUNs and to provide an

example representation of a

CANYUN GENRE with all

accompanying source data.

Although Nissle has significant 
clinical relevance and is similar to $E$. coli $\mathrm{K}-12$, there are no published Nissle metabolic network reconstructions that currently exist and are freely available. Nissle is a probiotic strain that has demonstrated measurable impacts on colonization resistance against human gastrointestinal pathogens (28-30). Additionally, it is important to note that several studies demonstrate that the metabolism of Nissle is phenotypically different from K-12. We generated novel phenotypic growth data for $E$. coli

320 Nissle using Biolog Phenotype MicroArray 96-well growth plates. We performed growth assays for the 321 carbon source plates, PM1 and PM2A, in both aerobic and anaerobic growth conditions. We found that 322 the metabolic consumption profile of Nissle is 9\% different from K-12 (Figure 8A and S2). There are 25 323 media conditions in which Nissle and K-12 do not align out of a total of 285. Nissle is able to grow in 16 324 conditions in which K-12 is not, in the other 9 conditions K-12 grows when Nissle does not. All 325 inconclusive results for K-12 were treated as no growth conditions. Data for K-12 anaerobic growth in 326 the PM2A plate does not exist on Ecocyc. All data is displayed in the Supplement (Figure S1 \& S2 and 327 Table S1).

We generated a CANYUN GENRE using the same input data discussed in Figure 1, although with 329 the Nissle specific annotation evidence and our set of phenotypic growth experiments. The final model that we generated had an overall accuracy of $92 \%$, with no false positive error. There are 18 false

331 negative conditions that could be fixed by adding organism-specific reactions to the model. There were 332481 reactions that received CVs and had high genetic evidence, indicating that they are likely to be 333 actively catalyzed by Nissle during exponential growth. Additionally, in the process of building the Nissle 334 model, we identified 114 reactions that have low genetic evidence, yet were included in the model with 335 CVs greater than zero. There were an additional 5 spontaneous reactions included. We assessed CVs associated with the 114 low evidence reactions to determine their rank of importance for future curation of the datasets used to generate this model. There were 34 reactions within the set that have no reference sequences and thus have unknown genetic evidence (Figure 8D). Finally, there were 80 
bioRxiv preprint doi: https://doi.org/10.1101/2021.08.13.456222; this version posted August 13, 2021. The copyright holder for this preprint (which was not certified by peer review) is the author/funder, who has granted bioRxiv a license to display the preprint in perpetuity. It is made available under aCC-BY 4.0 International license.

339 reactions that had both low genetic evidence and CVs greater than zero (Figure 8E). The reactions with

340 bitscores closer to 500 and high CVs have a high cumulative evidence indicating that they should be

341 assessed further to determine the appropriate reference sequences that should be added to the dataset

342 used to generate this model. 
A

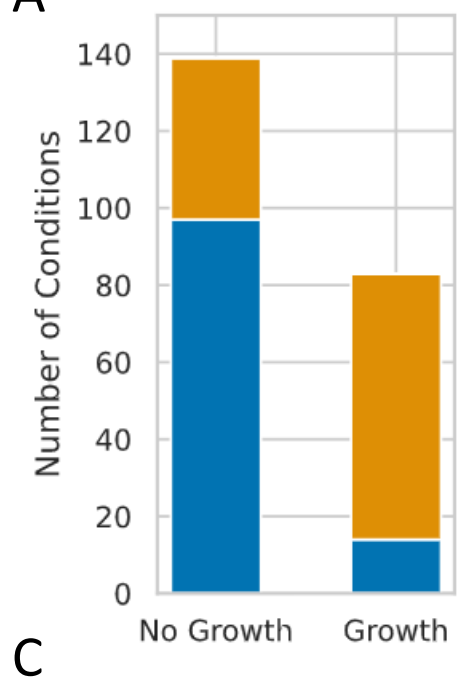

Universal Rxns

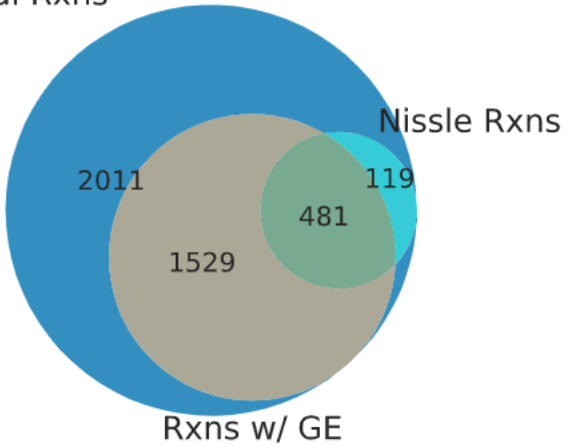

CANYUN reactions with GE

CANYUN reactions without GE

Reactions with GE alone

Universal Biochemical reactions

B

\begin{tabular}{ll}
$\begin{array}{l}\text { Predicted } \\
\text { Growth }\end{array}$ & $\begin{array}{l}\text { Predicted No } \\
\text { Growth }\end{array}$ \\
\hline
\end{tabular}

\begin{tabular}{lcc}
\hline Exp. & True Positive & False Negative \\
Growth & 65 & 18
\end{tabular}

Exp. No False Positive True Negative Growth $\quad 139$

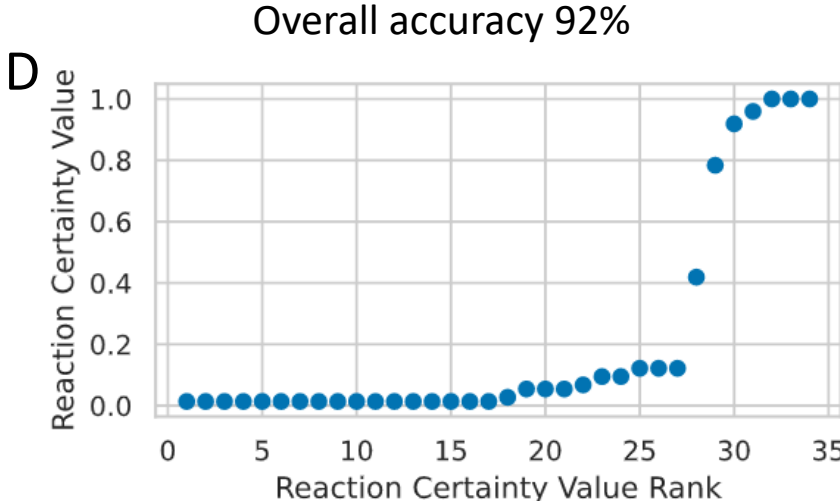

$\mathrm{E}$

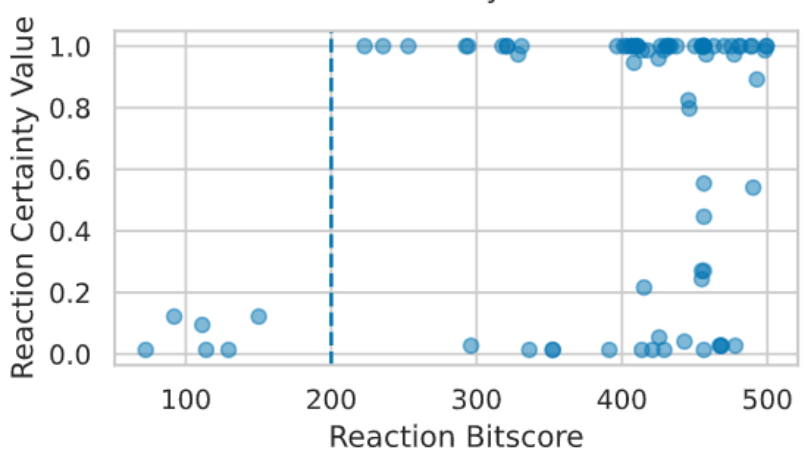

Figure 8: E. coli Nissle Model. A) Phenotypic data used to build the model. B) The final accuracy of the model is $92 \%$ with no false positive predictions. C) Model reaction classes. The sequence-to-reaction dataset used to generate genetic evidence bitscores includes 3,017 reactions with associated sequences. D) Reactions that receive certainty values, but do not have reaction bitscores. E) Reactions with Certainty Values and low bitscores (below 500). There are 80 reactions represented on this plot. Reactions with a high Certainty Value and a bitscore above 200 are likely candidates for additions to the sequence-to-reaction database.

\section{Discussion}

Introduced here is a procedural network reconstruction method for the generation of CANYUN 
dgFBA, to direct flux through the universal metabolic network during model building and curation resulting in a GENRE that is structurally different from manually curated GENREs. Cumulative evidence for inclusion of a given reaction in a CANYUN GENRE is explicitly quantified during the network reconstruction process. Existing methods rely heavily on genetic data to estimate the metabolic capabilities of an organism. CANYUNs fills a separate niche; it produces procedurally generated GENRES that include functional data such as phenotypic growth data as an integral step in the curation protocol. Maintaining a strong connection with all source data allows CANYUNs to guard against information loss that can occur. Most importantly, a core objective of CANYUNs is to leverage the uncertainty innate to the biological data used during the reconstruction process to generate a GENRE built upon continuous data inputs. This aspect of CANYUN GENREs differs from the presence or absence of reactions in other GENREs. The structure of CANYUN GENREs allows for the uncertainty across biological data to be managed via redundancy. Each type of data provides various benefits while mitigating associated error.

With some exceptions, GENREs are frequently referred to in the literature as either a network reconstruction or a model depending upon the context in which it is utilized. CANYUNs formalizes a structure that highlights the important differences between a network reconstruction and a model.

364 the evidence that is utilized to identify organism-specific reactions. A CANYUN GENRE can be viewed as 365 a model when utilizing only the reactions that acquire CVs. The conceptual framework underlying this distinction is grounded in the idea that phenotypic growth data should be utilized to contextualize the

367 genetic and biochemical data, rather than determine the absolute inclusion or exclusion of reactions 368 from a GENRE. By accepting that an organism-specific GENRE is simply a contextualized version of the 369 underlying universal metabolic network, there is additional flexibility that can be leveraged for future 370 curation of the GENRE with additional biological data or expansion of the source data. This concept is 371 the core difference between CANYUN GENREs and other GENREs generated using existing methods. 
The technical characteristics that make a CANYUN GENRE unique from other methods revolve

373 around a consistent connection to source data and management of associated uncertainty in the source

374 data. CANYUN GENREs are structured to facilitate future curation by ensuring that all source data is an

375 integral part of the model. As seen in Figure 8C, a CANYUN GENRE consists of four classifications of

376 reactions: Universal biochemical reactions, reactions with genetic evidence and no CV, reactions with

377 genetic evidence and a CV, and reactions with a CV and no genetic evidence. Each class of reaction has

378 an associated continuous spectrum that indicates how much evidence has contributed to the reaction

379 being in that class. Universal biochemical reactions have a spectrum of reference sequences (Figure 1D).

380 Reactions with only genetic evidence have the reaction bitscore which is positioned on a continuous

381 spectrum. Reactions with only a CV have the magnitude of the CV that represents the cumulative

382 phenotypic and biochemical evidence associated with the reaction. Finally, reactions with a CV and

383 genetic evidence have the most complex array of associated evidence including: genetic, phenotypic,

384 and biochemical.

Procedural generation methods benefit from existing manually curated GENREs via their

contribution to the universal biochemical network and the associated sequence-to-reaction reference

387 dataset. Manually curated versions of foundational data provide the base on which procedural

388 generation methods can be built upon. It has been shown that procedurally generated GENREs benefit

389 from manually curated data inputs (10). For example, ensuring that all reactions in the universal

390 biochemical network are mass-balanced and that there are no mass-generating loops in the network

391 eliminates the need for further thermodynamic-based curation of the resulting GENREs $(10,31)$. The high

392 specificity required for the annotation of metabolic enzymatic function with accompanying

393 thermodynamic and stoichiometric directionality is relatively unique to GENREs and thus a limiting

394 factor in the building process. Reaction directionality is a simple, yet important aspect of curating

395 GENREs. Often sequence annotation databases do not include specific information about reaction 
directionality. Directionality can become particularly important when a reaction is thermodynamically unfavorable in a certain direction. Improper directionality assignments can lead to free mass-generation and improper assignment of catalytic function. The CANYUNs method provides a way to quantify reaction evidence specific to directionality by calculating CVs specific to the direction of the flux through reactions. This level of specificity provides more control over the behavior of an organism specific network.

Genetic data is the base on which GENREs are built, yet not all genetic information is required to represent the metabolic network for an organism. Due to gene regulation and other aspects of in mind that genetic data, with all associated uncertainties, is simply an imperfect lens through which an organism specific model can begin to take shape. Functional phenotypic data, when paired with a

407 stoichiometrically accurate universal metabolic network, provides information for contextualizing the underlying genetic data. This conceptual framework provides the flexibility required for passively

409 allowing unknown gene regulation across differing growth conditions to influence the building process.

410 The core assumption in this conceptual model is that thermodynamic efficiency, both stoichiometric and

411 enzymatic, is the primary governing objective at the cellular level. This objective is technically achieved

412 during the prediction of growth by utilizing only the reactions with CVs for flux balance analysis. All

413 other reactions with genetic evidence alone did not demonstrate their activity during the training of the 414 model and are thus not active for growth predictions.

417 to the curation of specific models. The curation of specific models, separate from the source datasets, 418 can result in thermodynamic inconsistences among models that make it difficult to simulate metabolic interactions. This method lays the groundwork for data-driven expansion of the sequence-to-reaction 
dataset by quantifying phenotypic evidence for the inclusion of sequences slightly below the functional

421 bitscore threshold (set to 500 in this study). With enough phenotypic data from an array of organisms, it

422 would be possible to conservatively expand the reference dataset to propagate well-defined functional

423 annotations to many more sequences and thus expand the ability to generate accurate models across a

424 wide array of organisms. Additionally, CANYUN GENREs optimize for false negative predictions, thus

425 specifically identifying areas of the universal biochemical network that need manual additions. Moving

426 forward, CANYUNs may provide an additional starting point for analyses that provide more predictive

427 capabilities. Nevertheless, CANYUNs provides solutions for several challenges in expanding genome-

428 scale metabolic network reconstructions to model the vast array of microbes that exist in human-

429 associated microbial communities.

\section{$430 \quad$ Methods}

$431 \quad$ Universal metabolic network curation:

432 We started with the CarveMe universal model and added any new reactions from iML1515 to a

433 universal metabolic network. Any metabolites with multiple formulas were altered to maintain only the

434 metabolite formula used in the iML1515 model. Metabolites with multiple formulas that are not in the

435 iML1515 model were adjusted based on stoichiometric consistency across all reactions. The final

436 universal metabolic network is available on Github.

We utilized the following optimization problem to determine if the universal metabolic network

439 of sink reactions that allow flux to leave the system from within the cellular or periplasm compartment.

440 No metabolites are allowed to enter the system through exchange reactions. This algorithm provides an

441 output of all reactions that are able to carry flux when no external metabolites are provided. The

442 simultaneous maximization of flux through all sink reactions allows for a thorough evaluation of all

443 possible mass generating loops. 
Free mass generation check optimization problem

445

Maximize: $\sum \overrightarrow{v_{s n k}}$

446

subject to

$$
\overrightarrow{0} \leq \overrightarrow{v_{e x}} \leq \overrightarrow{0}
$$

$451 \overrightarrow{v_{\text {snk }}}=$ Sink $r x n$ flux vector

$452 \boldsymbol{S}=$ stoichiometric matrix

$453 \vec{v}=$ intracellular flux vector

$454 \overrightarrow{L B}=$ lower bound vector

$455 \overrightarrow{U B}=$ upper bound vector

$456 \overrightarrow{v_{e x}}=$ exchange rxn flux vector

457 CANYUNs Reconstruction Building Process:

We utilized the sequence-to-reaction database provided in the CarveMe publication. We aligned

459 the unknown protein sequence fasta file with Diamond to calculate sequence alignment bitscores for

460 each protein. We then calculated reaction bitscores for each reaction in the universal biochemical

461 network utilizing the CarveMe method. For the CANYUN GENRE construction, the superset of all flux-

462 carrying reactions determined using dgFBA for each of the known growth conditions are utilized to build

463 a draft network reconstruction. The draft network reconstruction is processed to determine all of the

464 conditionally essential reactions for each of the draft model growth predictions (true and false

465 positives). The reactions that are conditionally essential for more false positives than true positives are

466 reactions that can be used to improve predictive accuracy. The reaction with the most leverage to

467 improve model predictions is removed from the CANYUNs GENRE to create the final model. The model

468 building process requires roughly 30 minutes from start to finish on an Intel Xeon processor with 4

cores. 
CarveMe Model Generation:

471

We utilized CarveMe in a Windows 10 command line to generate a base model without

472 gapfilling and a gapfilled model with all known growth conditions. All default parameters were used

$473(10)$.

$474 \quad$ E. coli Nissle Data Collection and Model Generation:

475 We cultured E. coli Nissle in Biolog plates using a TECAN microplate reader. Optical density

476 measurements were performed using a 600 nanometer wavelength. We used Biolog PM1 and PM2A

477 plates. The cultures were started with an overnight culture in M9 4\% glucose medium at 37 degrees

478 Celsius from a single colony selection off an LB agar plate. The cells were centrifuged and washed with

479 PBS three times and finally resuspended and diluted into the base Biolog inoculation fluid. The resulting

480 OD of the Biolog inoculation fluid, after dilution, was calculated to be $0.01 \mathrm{OD}$. When cultured

481 anaerobically the OD of the plates were measured at the end of 40 hours shaking in an anaerobic

482 chamber. The baseline OD for each well was determined by filling a plate with the base media alone.

483 The OD of the aerobic plates was measured every 10 minutes for the whole time-course with shaking.

484 We acquired the Nissle genome from EMBL. With this genome we implemented CANYUNs to

485 calculate CVs and build an organism specific GENRE.

486 Code and Availability:

We utilized Python and Cobrapy for all aspects of this project. All code and data used is available 

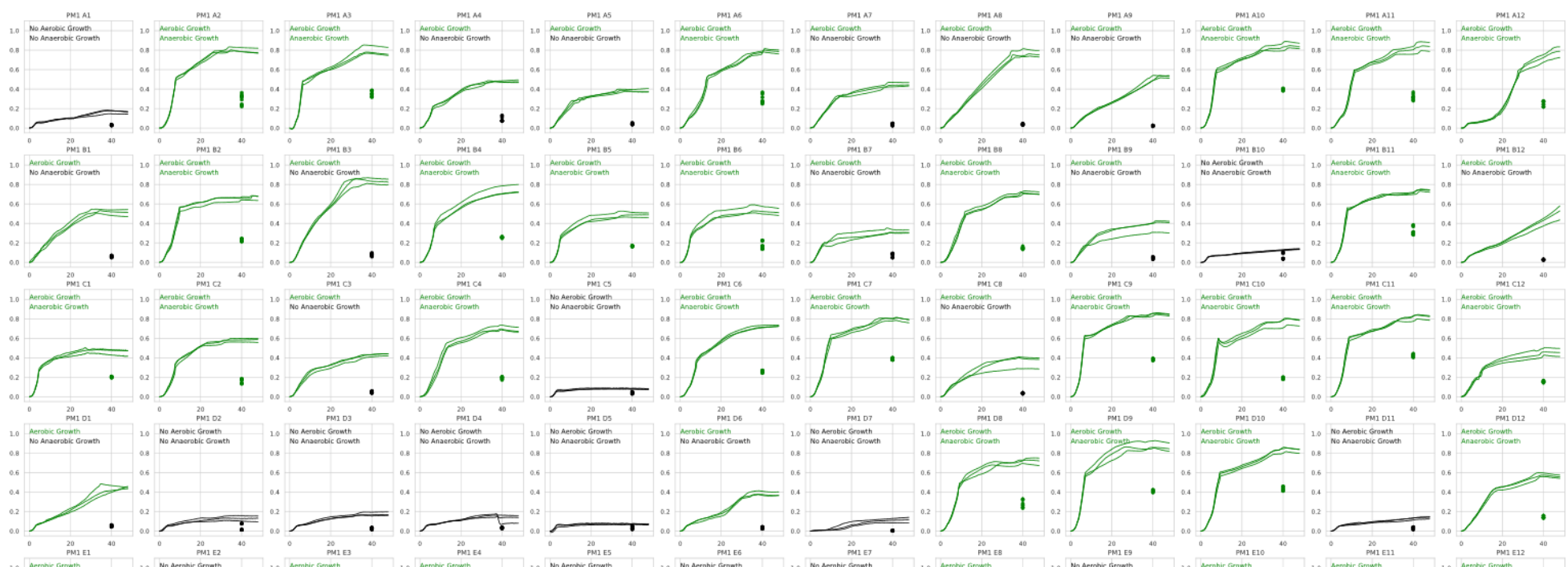

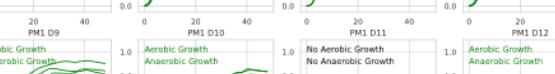

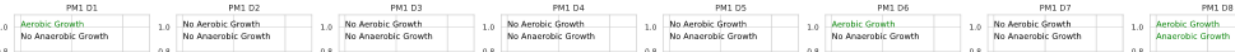

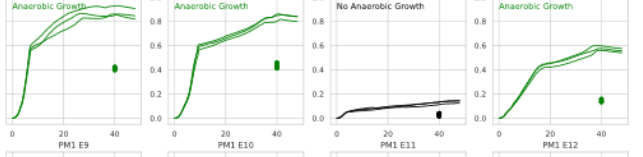

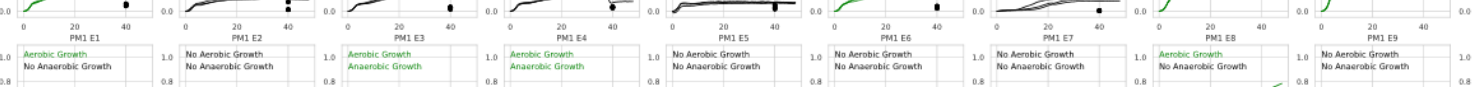

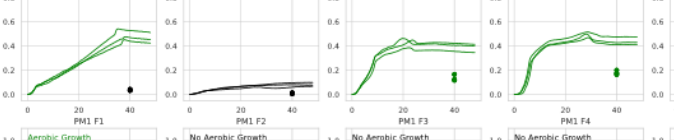

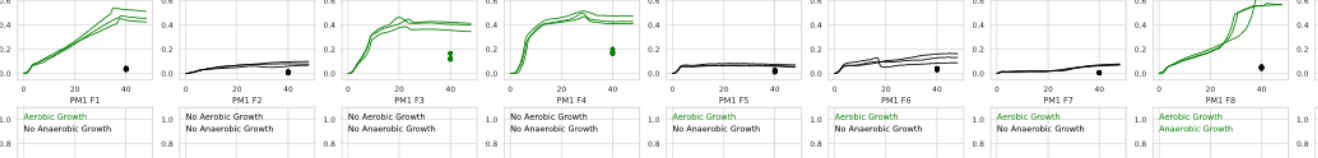
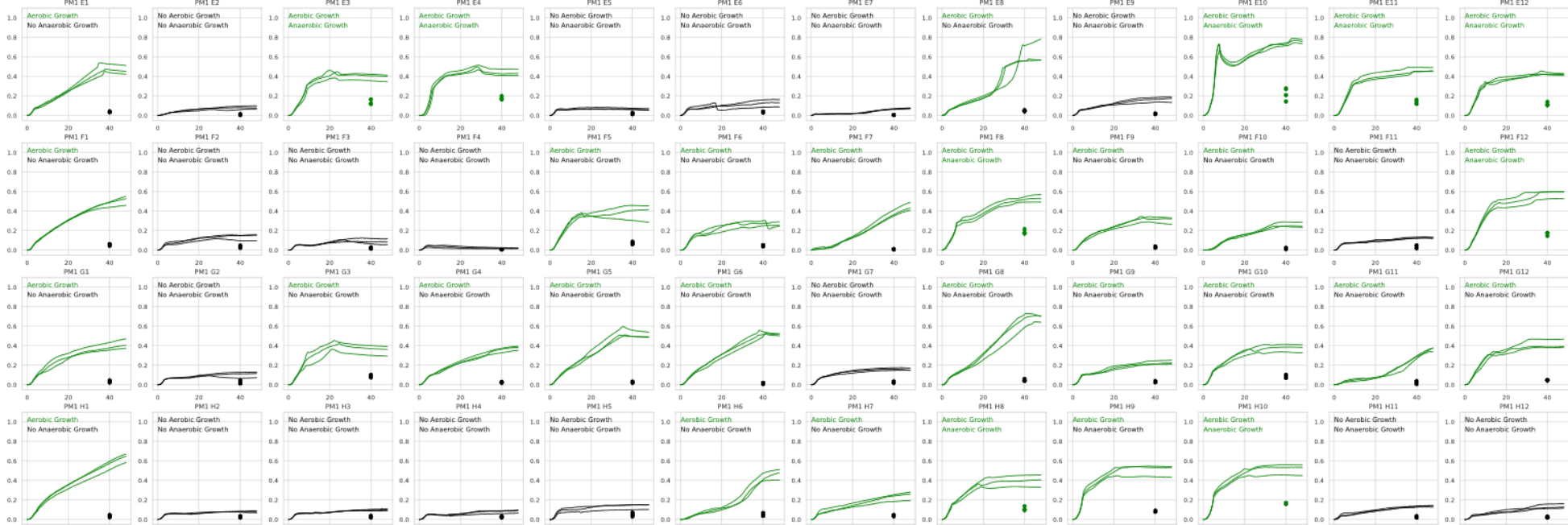


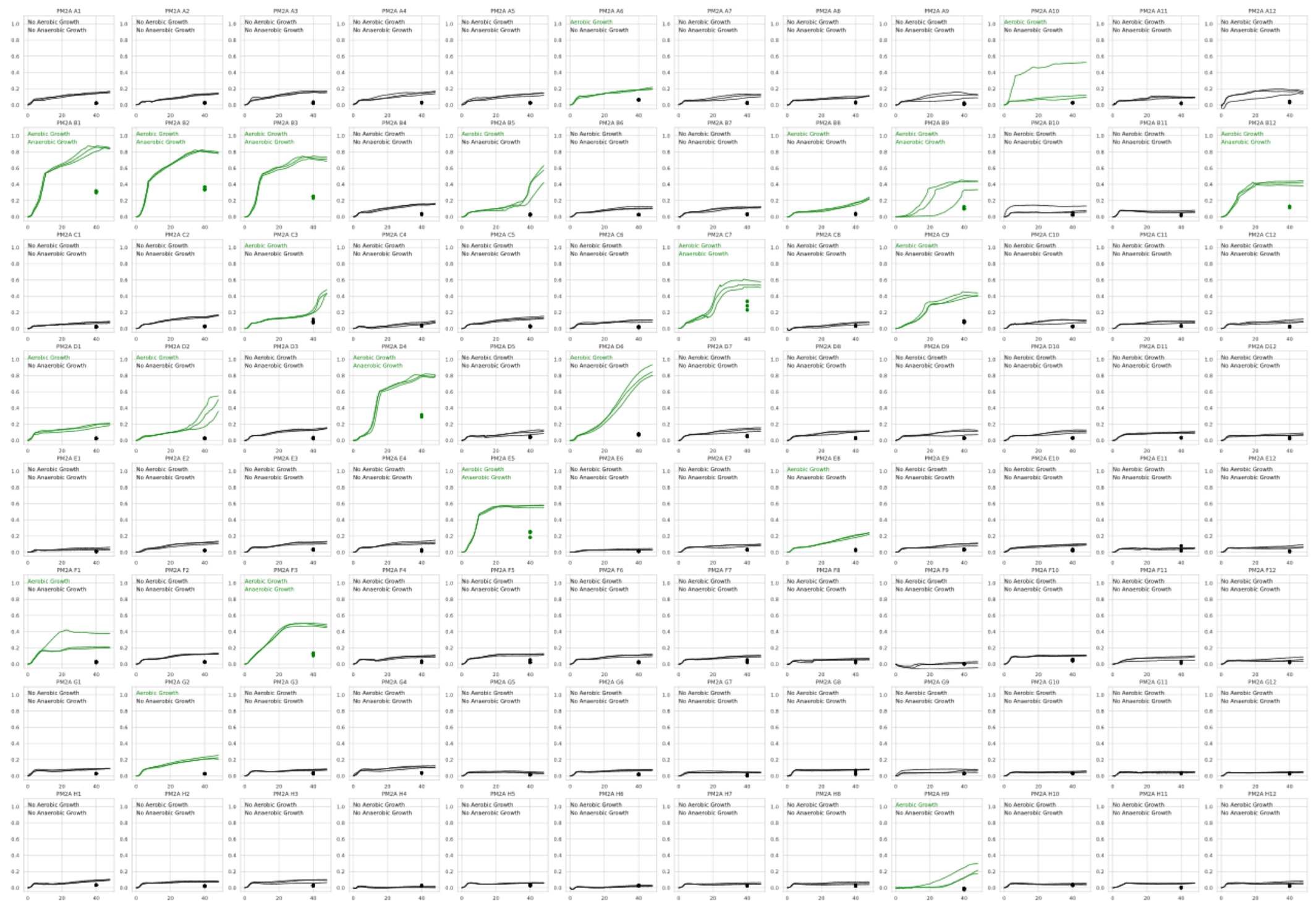

494 Figure S2: E. coli Nissle PM2A Biolog Growth Data. There are 22 aerobic growth conditions and 9 anaerobic growth conditions. 
497 Supplemental Table 1: Difference in E. coli Nissle growth compared to E. coli K12. Anaerobic data for K-12 growth in Biolog plate PM2A was not 498 available on EcoCyc.

\begin{tabular}{|c|c|c|c|c|c|}
\hline Index & Metabolite Name & Biolog Plate and Well \# & Culture Environment & E. coli K-12 Growth & E. coli Nissle Growth \\
\hline 1 & Dulcitol & PM1 - A12 & Aerobic & No Growth & Growth \\
\hline 2 & L-Glutamic acid & PM1 - B12 & Aerobic & No Growth & Growth \\
\hline 3 & Glucuronamide & $\mathrm{PM} 1-\mathrm{H} 7$ & Aerobic & No Growth & Growth \\
\hline 4 & N-Acetyl-D-Galactosamine & PM2A-B1 & Aerobic & No Growth & Growth \\
\hline 5 & D-Raffinose & PM2A - D1 & Aerobic & No Growth & Growth \\
\hline 6 & Salicin & PM2A - D2 & Aerobic & No Growth & Growth \\
\hline 7 & L-Alaninamide & $P M 2 A-G 2$ & Aerobic & No Growth & Growth \\
\hline 8 & D-Lactitol & $\mathrm{PM} 2 \mathrm{~A}-\mathrm{C} 3$ & Aerobic & No Growth & Growth \\
\hline 9 & L-Sorbose & PM2A - D4 & Aerobic & No Growth & Growth \\
\hline 10 & D-Arabinose & PM2A - B5 & Aerobic & No Growth & Growth \\
\hline 11 & D-Tagatose & PM2A - D6 & Aerobic & No Growth & Growth \\
\hline 12 & Arbutin & PM2A-B8 & Aerobic & No Growth & Growth \\
\hline 13 & $\beta$ - Hydroxybutyric acid & PM2A-E8 & Aerobic & No Growth & Growth \\
\hline 14 & 2-Deoxy- D-Ribose & PM2A - B9 & Aerobic & No Growth & Growth \\
\hline 15 & Laminarin & PM2A - A10 & Aerobic & No Growth & Growth \\
\hline 16 & M-Tartaric acid & PM1 - E2 & Aerobic & Growth & No Growth \\
\hline 17 & D-Fructose- 6-Phosphate & PM1-E4 & Aerobic & Growth & No Growth \\
\hline 18 & Acetoacetic acid & PM1 - G7 & Aerobic & Growth & No Growth \\
\hline 19 & $\alpha$ - Ketobutyric acid & PM1 - D7 & Aerobic & Growth & No Growth \\
\hline 20 & $\alpha$ - Hydroxybutyric acid & PM1-E7 & Aerobic & Growth & No Growth \\
\hline 21 & 5-Keto-D- Gluconic acid & PM2 - E12 & Aerobic & Growth & No Growth \\
\hline 22 & D-Serine & PM1 - B1 & Anaerobic & Growth & No Growth \\
\hline 23 & D-Saccharic acid & PM1-A4 & Anaerobic & Growth & No Growth \\
\hline 24 & L-Galactonic acid- $\gamma$ - Lactone & PM1-H9 & Anaerobic & Growth & No Growth \\
\hline 25 & Methylpyruvate & PM1-G10 & Anaerobic & Growth & No Growth \\
\hline
\end{tabular}




\section{References}

1. Clemente JC, Ursell LK, Parfrey LW, Knight R. The Impact of the Gut Microbiota on Human Health: An Integrative View. Cell. 2012 Mar 16;148(6):1258-70.

2. Galland L. The Gut Microbiome and the Brain. J Med Food. 2014 Dec;17(12):1261-72.

3. Gopalakrishnan V, Helmink BA, Spencer CN, Reuben A, Wargo JA. The Influence of the Gut Microbiome on Cancer, Immunity, and Cancer Immunotherapy. Cancer Cell. 2018 Apr 9;33(4):57080 .

4. Zhu B, Wang X, Li L. Human gut microbiome: the second genome of human body. Protein Cell. 2010 Aug 1;1(8):718-25.

5. Nicholson JK, Holmes E, Kinross J, Burcelin R, Gibson G, Jia W, et al. Host-Gut Microbiota Metabolic Interactions. Science. 2012 Jun 8;336(6086):1262-7.

6. Magnúsdóttir S, Heinken A, Kutt L, Ravcheev DA, Bauer E, Noronha A, et al. Generation of genomescale metabolic reconstructions for 773 members of the human gut microbiota. Nat Biotechnol. 2017 Jan;35(1):81-9.

7. Diener C, Gibbons SM, Resendis-Antonio O. MICOM: Metagenome-Scale Modeling To Infer Metabolic Interactions in the Gut Microbiota. mSystems. 2020 Jan 21;5(1):e00606-19.

8. Orth JD, Thiele I, Palsson B $\varnothing$. What is flux balance analysis? Nat Biotechnol. 2010 Mar;28(3):245-8.

9. Thiele I, Vlassis N, Fleming RMT. fastGapFill: efficient gap filling in metabolic networks. Bioinformatics. 2014 Sep 1;30(17):2529-31.

10. Machado D, Andrejev S, Tramontano M, Patil KR. Fast automated reconstruction of genome-scale metabolic models for microbial species and communities. Nucleic Acids Res. 2018 Sep 6;46(15):7542-53.

11. Mundy M, Mendes-Soares H, Chia N. Mackinac: a bridge between ModelSEED and COBRApy to generate and analyze genome-scale metabolic models. Bioinformatics. 2017 Aug 1;33(15):2416-8.

12. Seaver SMD, Liu F, Zhang Q, Jeffryes J, Faria JP, Edirisinghe JN, et al. The ModelSEED Biochemistry Database for the integration of metabolic annotations and the reconstruction, comparison and analysis of metabolic models for plants, fungi and microbes. Nucleic Acids Res. 2021 Jan 8;49(D1):D575-88.

13. Thiele I, Palsson $\mathrm{B} \emptyset$. A protocol for generating a high-quality genome-scale metabolic reconstruction. Nat Protoc. 2010 Jan;5(1):93-121.

14. Kumar VS, Maranas CD. GrowMatch: an automated method for reconciling in silico/in vivo growth predictions. PLoS Comput Biol. 2009;5(3). 
15. King B, Farrah T, Richards MA, Mundy M, Simeonidis E, Price ND. ProbAnnoWeb and ProbAnnoPy: probabilistic annotation and gap-filling of metabolic reconstructions. Bioinformatics. 2018 May 1;34(9):1594-6.

16. Schellenberger J, Park JO, Conrad TM, Palsson B $\emptyset$. BiGG: a Biochemical Genetic and Genomic knowledgebase of large scale metabolic reconstructions. BMC Bioinformatics. 2010;11(1):213.

17. Norsigian CJ, Pusarla N, McConn JL, Yurkovich JT, Dräger A, Palsson BO, et al. BiGG Models 2020: multi-strain genome-scale models and expansion across the phylogenetic tree. Nucleic Acids Res. 2020 Jan 8;48(D1):D402-6.

18. Schmidt BJ, Ebrahim A, Metz TO, Adkins JN, Palsson B $\varnothing$, Hyduke DR. GIM3E: condition-specific models of cellular metabolism developed from metabolomics and expression data. Bioinformatics. 2013 Nov 15;29(22):2900-8.

19. Jenior ML, Jr TJM, Dougherty BV, Papin JA. Transcriptome-guided parsimonious flux analysis improves predictions with metabolic networks in complex environments. PLOS Comput Biol. 2020 Apr 16;16(4):e1007099.

20. Gama-Castro S, Salgado H, Santos-Zavaleta A, Ledezma-Tejeida D, Muñiz-Rascado L, García-Sotelo JS, et al. RegulonDB version 9.0: high-level integration of gene regulation, coexpression, motif clustering and beyond. Nucleic Acids Res. 2016 Jan 4;44(D1):D133-43.

21. Monk JM, Lloyd CJ, Brunk E, Mih N, Sastry A, King Z, et al. iML1515, a knowledgebase that computes Escherichia coli traits. Nat Biotechnol. 2017 Oct 11;35(10):904-8.

22. Keseler IM, Mackie A, Santos-Zavaleta A, Billington R, Bonavides-Martínez C, Caspi R, et al. The EcoCyc database: reflecting new knowledge about Escherichia coli K-12. Nucleic Acids Res. 2017 Jan 4;45(D1):D543-50.

23. Keseler IM, Collado-Vides J, Santos-Zavaleta A, Peralta-Gil M, Gama-Castro S, Muñiz-Rascado L, et al. EcoCyc: a comprehensive database of Escherichia coli biology. Nucleic Acids Res. 2011 Jan;39(Database issue):D583-90.

24. González-Pech RA, Stephens TG, Chan CX. Commonly misunderstood parameters of NCBI BLAST and important considerations for users. Bioinformatics. 2019 Aug 1;35(15):2697-8.

25. Pearson WR. An Introduction to Sequence Similarity ("Homology") Searching. Curr Protoc Bioinforma. 2013;42(1):3.1.1-3.1.8.

26. Gibbons TR, Mount SM, Cooper ED, Delwiche CF. Evaluation of BLAST-based edge-weighting metrics used for homology inference with the Markov Clustering algorithm. BMC Bioinformatics. 2015 Jul 10;16(1):218.

27. Lewis NE, Hixson KK, Conrad TM, Lerman JA, Charusanti P, Polpitiya AD, et al. Omic data from evolved $\mathrm{E}$. coli are consistent with computed optimal growth from genome-scale models. Mol Syst Biol. 2010 Jul 27;6:390. 
28. Leatham MP, Banerjee S, Autieri SM, Mercado-Lubo R, Conway T, Cohen PS. Precolonized Human Commensal Escherichia coli Strains Serve as a Barrier to E. coli 0157:H7 Growth in the Streptomycin-Treated Mouse Intestine. Infect Immun. 2009 Jul 1;77(7):2876-86.

29. Altenhoefer A, Oswald S, Sonnenborn U, Enders C, Schulze J, Hacker J, et al. The probiotic Escherichia coli strain Nissle 1917 interferes with invasion of human intestinal epithelial cells by different enteroinvasive bacterial pathogens. FEMS Immunol Med Microbiol. 2004 Apr 1;40(3):223-9.

30. Maltby R, Leatham-Jensen MP, Gibson T, Cohen PS, Conway T. Nutritional Basis for Colonization Resistance by Human Commensal Escherichia coli Strains HS and Nissle 1917 against E. coli O157:H7 in the Mouse Intestine. PLOS ONE. 2013 Jan 17;8(1):e53957.

31. Fritzemeier CJ, Hartleb D, Szappanos B, Papp B, Lercher MJ. Erroneous energy-generating cycles in published genome scale metabolic networks: Identification and removal. PLOS Comput Biol. 2017 Apr 18;13(4):e1005494. 